\title{
Prototype de traqueur solaire à deux axes
}

\author{
Fabrice Robert, Jean-Paul Bécar*, Jean-Louis Alexief, Jean-Charles Canonne*, Laurent Vermeiren**, \\ *LAMAV FR-CNRS 2956, **LAMIH UMR CNRS 8530, \\ \{ fabrice.robert, jean-paul.becar, jean-louis.alexief, jean-charles.canonne,laurent.vermeiren\}@univ- \\ valenciennes.fr \\ IUT GEII, Le Mont-Houy 59313 Valenciennes Cedex 9
}

\begin{abstract}
RÉSUMÉ : Ce document relate une partie achevée d'un projet pluridisciplinaire de conception, réalisation, expérimentation et automatisation d'un prototype de maquette de traqueur solaire à deux axes. Initié à son origine par des questionnements d'étudiants sur les énergies renouvelables et plus particulièrement sur l'énergie solaire, le projet a rassemblé des enseignants et des étudiants autour de leurs compétences académiques et personnelles. L'électronique embarquée, l'automatique et les mathématiques, la gestion de projets en sont quelques-unes. L'accueil d'étudiants finlandais spécialistes du domaine du génie électrique et d'élèves ingénieurs en alternance a alimenté l'édifice. Parallèlement à ces travaux, d'autres étudiants du domaine de la conception mécanique assistée par ordinateur ont effectué une étude de faisabilité d'un traqueur solaire autonome à deux axes supportant un panneau solaire d'un mètre carré maximum. Le schéma du support est proposé en fin d'article. Sa réalisation par des étudiants spécialistes en chaudronnerie est en cours. Une telle aventure humaine scientifique et technique a été rendue possible à la fois par les acteurs mais aussi grâce à la plateforme technologique de l'Institut Universitaire de Technologie qui abrite le matériel et les logiciels adéquats. Les étudiants et les enseignants ont pu ainsi motiver à leur tour leurs camarades et leurs collègues au fil des ans.
\end{abstract}

Mots clés : modélisation mathématique, mesures, instrumentation, pédagogie par projet, outils logiciels, mesure du temps, modèle de Ptolémée, équation du temps, énergies renouvelables, microcontrôleur.

\section{INTRODUCTION}

Les énergies renouvelables constituent aujourd'hui un thème d'actualité porteur d'une image novatrice auprès des étudiants. Elles les motivent par l'émergence d'un vivier d'emplois en adéquation avec l'actualité, leur goût et leur formation académique. C'est l'intérêt des étudiants pour ces énergies, et plus particulièrement sur l'énergie solaire photoélectrique qui a nourri le projet. Leur questionnement a rencontré de nombreux échos à la fois auprès d'autres étudiants mais aussi des enseignants sollicitant ainsi une grande partie du potentiel humain de l'IUT. Le génie électrique, le génie mécanique, l'automatique et les mathématiques représentent le socle pluridisciplinaire du projet. La plateforme technologique du département est ainsi devenue un outil de ressources incontournable pour concevoir, réaliser, expérimenter et automatiser un prototype de traqueur solaire à deux axes.

L'objectif final est la réalisation future d'un traqueur solaire autonome à deux axes devant supporter un panneau solaire d'un poids maximum de vingt kilos. Il sera actionné par deux servomoteurs. Les moteurs seront commandés de sorte que le panneau solaire puisse à tout instant suivre la course du soleil dans le ciel afin d'être dans le maximum de flux lumineux. La partie mécanique a été confiée aux étudiants de la licence professionnelle de conception mécanique assistée par ordinateur de l'IUT. Il en est resulté les schémas fonctionnels, les plans du prototype et les coûts de l'étude et de sa réalisation. La partie électronique est décrite dans le document. De plus, pour entretenir la motivation des étudiants, le travail interdisciplinaire a égale- ment fait l'objet d'une participation à un concours international sur les énergies renouvelables [1]. Les présents travaux décrivent la réalisation, et l'exploitation d'une maquette de traqueur solaire. Outre la partie mécanique réalisée par une société de robotique, quelques éléments nécessaires à sa réalisation en vraie grandeur figurent en annexe de ce papier.

L'article décrit alors les étages de transformation des paramètres utiles comme les calculs astronomiques, les cartes d'acquisition, la carte et le programme de commande des servomoteurs. La section 2 fournit une description du schéma général du système. La section 3 décrit la maquette du traqueur solaire. L'électronique embarquée, les caractéristiques techniques et la programmation du microcontrôleur y sont données pour pouvoir être reproduites. Elle relate les travaux actuels des groupes d'étudiants en adéquation avec leur compétence technique récemment acquise. La section 4 tire un certain nombre de conclusions et propose des perspectives de développement.

En annexe, figurent le câblage du module de commande des moteurs et son programme rédigé en langage $\mathrm{C}$. L'article est rédigé de sorte que les sections présentent un étage de conception ou de réalisation $d u$ prototype. Elles indiquent également la participation des étudiants au projet en précisant leur cursus afin de pouvoir apprécier le niveau de compétence requis.

\section{SCHEMA DU TRAQUEUR SOLAIRE}

Le synoptique général de la maquette du traqueur solaire à deux axes est donné en figure 1 . Il se compose de quatre étages. 
A partir de la latitude et la date, le premier étage fournit au système les coordonnées locales du soleil mesurées en degrés. Ces dernières représentent les entrées du second étage du dispositif. Il renvoie les valeurs en tension correspondante aux angles d'azimut et d'élévation. La relation choisie est linéaire pour une mise en œuvre rapide.

Le troisième étage concerne la partie électronique embarquée. La carte électronique commande les servomoteurs par Modulation de Largeur d'Impulsion ou Pulse Width Modulation en anglais à partir des tensions représentatives de l'azimut et de l'élévation locales Le dernier étage est la partie mécanique du support. Il possède un mât vertical sur lequel sont fixés les deux servomoteurs qui déplacent le panneau solaire en azimut et élévation. Un bornier est placé à la base du mât pour assurer la commande. Le prototype final a été réalisé en deux exemplaires pour des raisons de dimensionnement des cellules photovoltaïques.

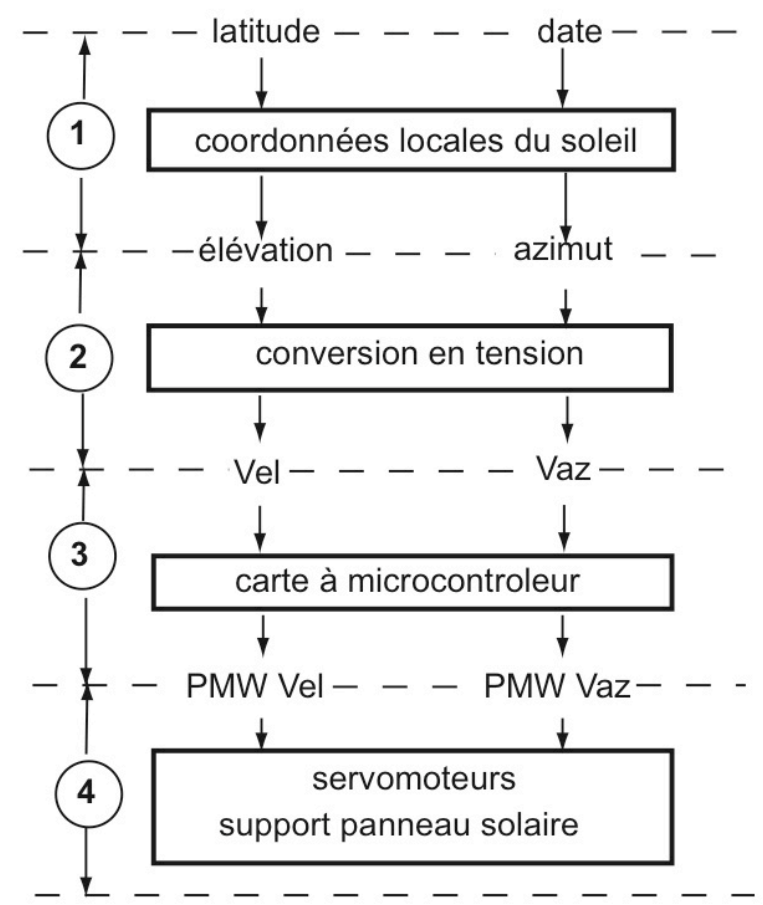

Fig 1. Synoptique du prototype de traqueur solaire deux axes.

Dans la suite sont décrits les étages 1 et 2 du traqueur. L'étage 1 (Figure 1) traite du calcul de ces coordonnées locales du soleil. L'algorithme est basé sur le modèle de Ptolémée et les relations de Gauss pour les coordonnées sphériques. Le modèle de Ptolémée a été choisi d'une part pour la facilité d'implémentation des calculs dans le microcontrôleur et d'autre part pour la précision suffisante dans ce cas. Cette partie a fait l'objet de plusieurs exposés et publications non repris ici $[2,3]$. Les algorithmes de calcul des coordonnées ont d'abord été écrits sous le logiciel Maple dédié au calcul scientifique et doté de nombreuses fonctions de représentation graphique. Pratiqué par les étudiants de première et deuxième année GE2I dans le cadre des enseignements de mathématiques, le logiciel a servi au calcul et la représentation d'éphémérides qui ont permis une vérification avec les sites officiels de calcul astronomiques comme par exemple $w w w$.imcce.fr. Ils ont ensuite été réécrits en langage $\mathrm{C}$ pour pouvoir d'une part être appliqués à un outil de développement industriel et d'autre part être installés dans un nouveau microcontrôleur.

La tâche de conversion a été confiée à un groupe d'étudiants finlandais lors d' un stage à l'IUT pour une durée de trois mois dans le cadre d'échanges européens Erasmus. Le but visé ici est de faire travailler ces étudiants sur un environnement industriel et un logiciel de développement associé comme Labview et de collaborer avec d'autres étudiants élèves ingénieurs en alternance.

\begin{tabular}{|l|l|l|}
\hline Angle Rotation & Tension Vaz & $\begin{array}{l}\text { Largeur d'impul- } \\
\text { sion }\end{array}$ \\
\hline$-90^{\circ}$ & $0 \mathrm{~V}$ & $0.5 \mathrm{~ms}$ \\
\hline $0^{\circ}$ & $2.5 \mathrm{~V}$ & $1.5 \mathrm{~ms}$ \\
\hline$+90^{\circ}$ & $5 \mathrm{~V}$ & $2.5 \mathrm{~ms}$ \\
\hline
\end{tabular}

Tab 1. Tableau des conversions

Les travaux des étudiants finlandais serviront de base à la rédaction d'un mémoire technique validant leur diplôme bachelor of science. L'expérience a montré par ailleurs que ce type de stage constitue un élément non négligeable dans la recherche d'un premier emploi.

Le programme en langage $C$ prend pour entrée la latitude du lieu et la date. Il renvoie les coordonnées locales du soleil soit l'azimut et l'élévation. L'azimut varie de $-90^{\circ}$ à $+90^{\circ}$. L'élévation varie dans l'intervalle $0 . .90^{\circ}$. Les valeurs sont converties en tensions de l'intervalle $0 . .5 \mathrm{~V}$ de manière linéaire respectivement (voir table 1).

L'outil de développement est constitué d'un contrôleur embarqué NI-PXI-8196, d'une carte d'acquisition NIPXI-6221 dans un rack PXI-1042 le tout piloté par Labview.

Pour pallier l'électronique lourde mise en œuvre, il est envisagé de réaliser une carte à microcontrôleur dont la fonction sera de proposer une saisie au clavier de la latitude et de la date et un contrôle par afficheur. Le programme interne sera chargé de calculer les coordonnées locales du soleil et d'assurer les conversions en tensions analogiques de l'azimut et l'élévation. Ces dernières pilotent les servomoteurs par l'intermédiaire d'une carte à microcontrôleur décrite dans la section suivante. 


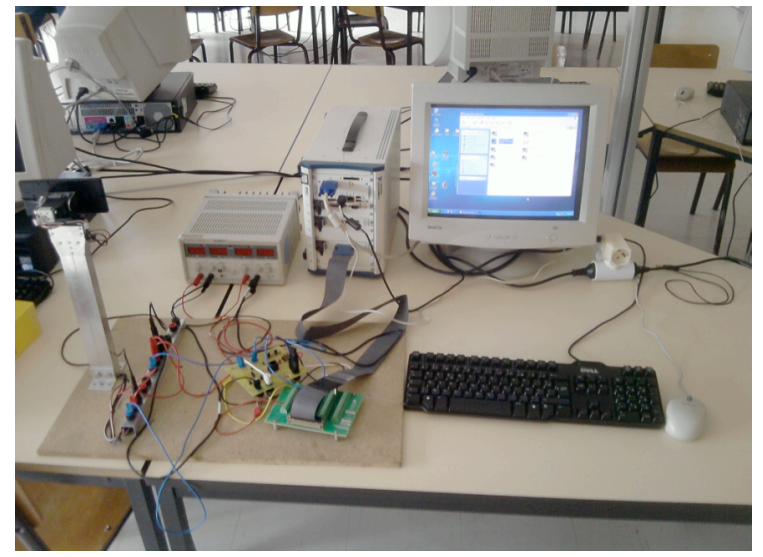

Fig 2. Electronique de commande du traqueur

\section{CARTE DE COMMANDE DES SERVO- MOTEURS}

Cette section décrit l'étage 3 du prototype de la figure 1. Le travail a été effectué dans le cadre de la formation des étudiants de deuxième année de la spécialité génie électrique. Le projet technique a concerné 12 étudiants encadrés par un enseignant d'électronique. L'enseignant a réalisé la conception globale matérielle et logicielle. Les étudiants ont effectué la conception détaillée, la réalisation, la programmation et les tests. Les traces du travail et la documentation technique ont été consignées dans un rapport final.

La section décrit la mise en œuvre de la carte de commande des servomoteurs. En entrée, les tensions analogiques comprises entre 0 et $5 \mathrm{~V}$ représentant l'azimut et l'élévation sont notées Vaz et Vel respectivement. En sortie, les signaux Saz et Sel pilotent les servomoteurs. Ils sont du type Pulse Width Modulation -PWM- de fréquence $50 \mathrm{~Hz}$ et de rapport cyclique variable. La période est de $20 \mathrm{~ms}$ et la largeur de l'impulsion détermine l'angle de rotation du servomoteur.

L'unité de traitement assure les fonctions de conversion analogique numérique des entrées, le calcul de la largeur d'impulsion et l'élaboration des signaux PWM Saz et Sel.

Le module SERVO1 est conçu autour d'un microcontrôleur Pic 16F684 de Microchips L'oscillateur interne à $8 \mathrm{MHz}$, le convertisseur analogique numérique et les timers figurent parmi les ressources utilisées. Les entrées Vaz et Vel passent par un filtre passe bas dont la fréquence de coupure est d'environ $70 \mathrm{~Hz}$. Un écrêtage de ces entrées est également prévu. Les essais ont montré qu'il était nécessaire de séparer les alimentations du microcontrôleur et des servomoteurs, pour obtenir un fonctionnement stable sans oscillations.

Le programme du microcontrôleur est développé en langage $\mathrm{C}$ dans l'environnement MPLAB de Microchips. Il consiste en un cycle de $20 \mathrm{~ms}$ dans lequel trois tâches sont réalisées successivement : l'acquisition des signaux d'entrées, le calcul de la largeur d'impulsion et la restitution des signaux PWM. Le timer1 est utilisé pour le cycle de $20 \mathrm{~ms}$ et le timer0 pour la largeur d'impulsion. Des butées logicielles ont été incluses de façon à limiter les rotations par rapport aux possibilités de la mécanique de suivi.

En annexe sont donnés le brochage du microcontrôleur, la fonction en $\mathrm{C}$ donnant la conversion des signaux .

\section{TRAQUEURS SOLAIRES 2 AXES}

Cette partie présente 2 prototypes de supports de panneaux solaires photovoltaïques. Les 2 premiers réalisés fournissent ainsi une base de travail pour la mesure de performances de panneaux solaires et l'installation d'un système de régulation. Le but en est l'optimisation de l'énergie rendue au système.

Le premier prototype (fig. 3 avant plan) se compose d'un pied en aluminium d'une hauteur de $30 \mathrm{~cm}$ fixé sur une platine assurant la stabilité. La partie supérieure comporte les deux servomoteurs du type FUTABA S300 avec retour d'information. Ils développent chacun un couple de $3.2 \mathrm{Kg} . \mathrm{cm}$ sous $4.8 \mathrm{~V}$ et $4.1 \mathrm{Kg} . \mathrm{cm}$ sous $6 \mathrm{~V}$ pour un poids de 37.2 grammes. Ils animent un panneau solaire polycristallin d'une puissance de $0.6 \mathrm{~W}$ de dimensions $140 \times 67 \times 8 \mathrm{~mm}$ qui fournit une tension de $2 \mathrm{~V}$ et pèse 150 grammes.

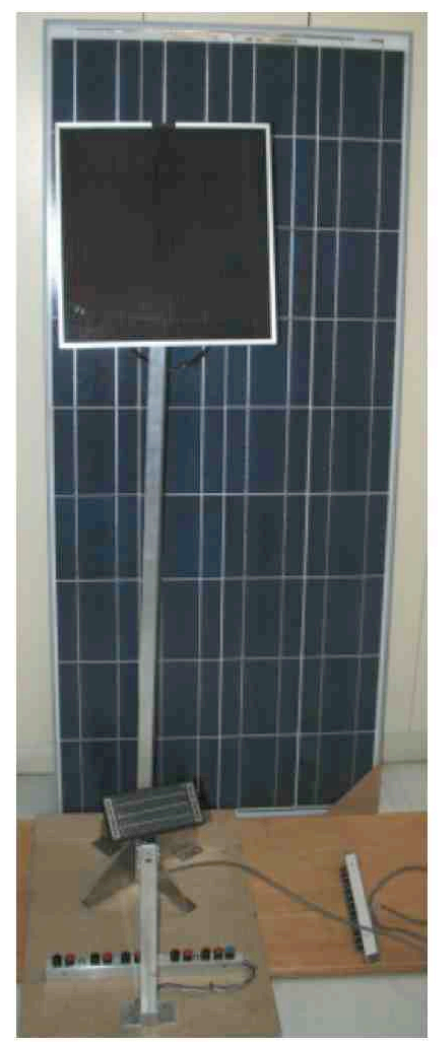

Fig 3. Prototypes de traqueur

Le second prototype (fig. 3 second plan) se compose d'un pied en aluminium d'une hauteur de $100 \mathrm{~cm}$ fixé sur une platine assurant la stabilité. La partie supérieure 
comporte les deux servomoteurs FUTABA S3305. Ils développent chacun un couple de $8.9 \mathrm{Kg} . \mathrm{cm}$ sous $6 \mathrm{~V}$ pour un poids de 46.5 grammes. Les moteurs animent un panneau solaire en silicium amorphe d'une puissance de $4 \mathrm{~W}$ de dimensions $315 \times 315$ qui fournit une tension de $2 \mathrm{~V}$ et pèse 750 grammes. La commande des servomoteurs est établie depuis l'environnement industriel. Pour compléter les travaux sur les maquettes, des étudiants de la licence professionnelle de conception mécanique assistée par ordinateur ont réalisé une étude de faisabilité d'un traqueur solaire autonome.

Il représente l'étage 4 de la figure 1. Le cahier des charges du produit est ici de concevoir la partie mécanique d'un support de panneau solaire de dimensions 1476 × 659 × $35 \mathrm{~mm}$ pour un poids de $12.8 \mathrm{~kg}$ (Fig 3 arrière plan). La figure 4 est issue de l'étude de faisabilité développée du logiciel Catia

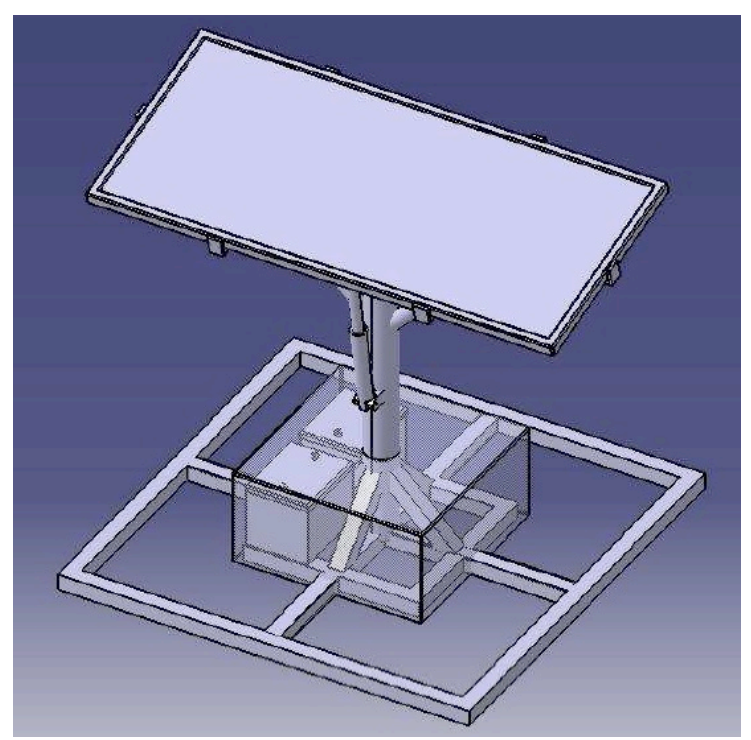

Fig 5. Support mécanique d'un panneau photovoltaique

\section{CONCLUSION ET PERSPECTIVES}

Ce document relate une partie achevée d'un projet pluridisciplinaire de conception, réalisation, expérimentation et automatisation d'un prototype de maquette de traqueur solaire à deux axes. L'environnement de travail pluridisciplinaire par la mise en commun des connaissances, internationales par l'accueil d'étudiants finlandais et industrielles par les projets d'élèves ingénieurs en alternance a enrichi le projet au fil des ans. Le papier donne les moyens de reproduire à moindre cout l'étage de commande des servomoteurs.

L'étage antérieur est développé sous un environnement industriel. Les coordonnées du soleil sont placées dans 6 fichiers pour les 12 mois de l'année. Le pilotage est effectué sous le logiciel Labview.

La future étude consiste à programmer un microcontrôleur capable d'effectuer des calculs mathématiques sur des nombres à virgule flottante afin de pouvoir disposer des valeurs des angles d'azimut et d'élévation. Les données d'entrée sont ici la latitude et la date. Les deux prototypes serviront de support d'étude à l'installation d'un régulateur flou pour minimiser la puissance consommée par les moteurs. Par ailleurs le modèle grandeur nature (Fig. 4) est en cours de réalisation pour la partie mécanique seule par un groupe d'étudiants d'un lycée du cursus conception et réalisation de carrosserie. Il se poursuit par l'expérimentation puis la mise en oeuvre d'algorithmes de recherche du point de puissance maximum d'un panneau solaire. Ces travaux sont entrepris par un groupe d'étudiants apprentis ingénieurs dans le cadre d'une initiation à la recherche.

Le panneau solaire supporté par la maquette sera asservi par un régulateur flou afin d'optimiser la fourniture d'énergie.

\section{Remerciements}

L'Institut Universitaire de Technologie de Valenciennes a mis à la disposition du groupe d'étudiants et d'enseignants une salle équipée d'ordinateurs et de logiciels adéquats, qu'il en soit ici vivement remercié.

Les remerciements s'adressent également aux étudiants de l'université des sciences appliquées du Satakunta de Pori en Finlande, de l'école d'ingénieurs Cesi d'Arras, et du lycée technique du Hainaut de Valenciennes.

\section{Bibliographie}

[1] Concours live-edge Janvier 2009 http://www.liveedge.com/en_US/Registration Number: 10560

[2] Bécar J.P., Canonne J.C., Robert F.,Vermeiren L., An example of scientific and technological project: the path of the sun IADAT Journal of Advanced Technology on Education-IJAT-e, Volume 3, Number 2, September 2007., pp 375-377, ISSN 1698-1073.

[3] Jean-Paul Bécar, Fabrice Robert, Jean-Charles Canonne, Laurent Vermeiren, Dispositif expérimental d'analyse des performances de cellules photovoltaïques J3eA Vol. 8 No. HORS SÉRIE 1 (2009) Special Edition: CETSIS 2008 http://www.j3ea.org/ ref 1009.

http://www.microchip.com/ http://www.futaba-rc.com/servos/servos.html http://www.easyrobotics.fr/

Annexes 


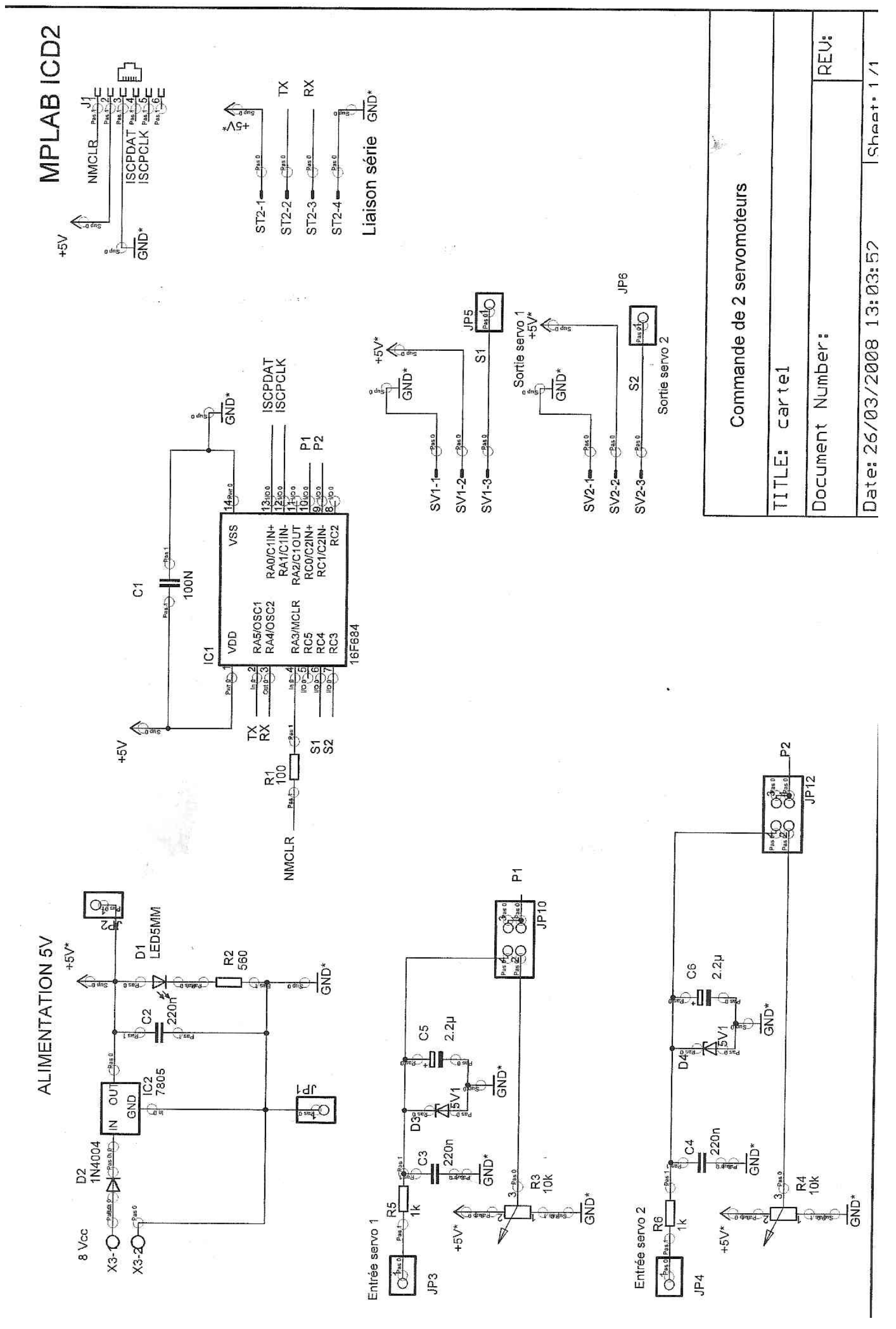




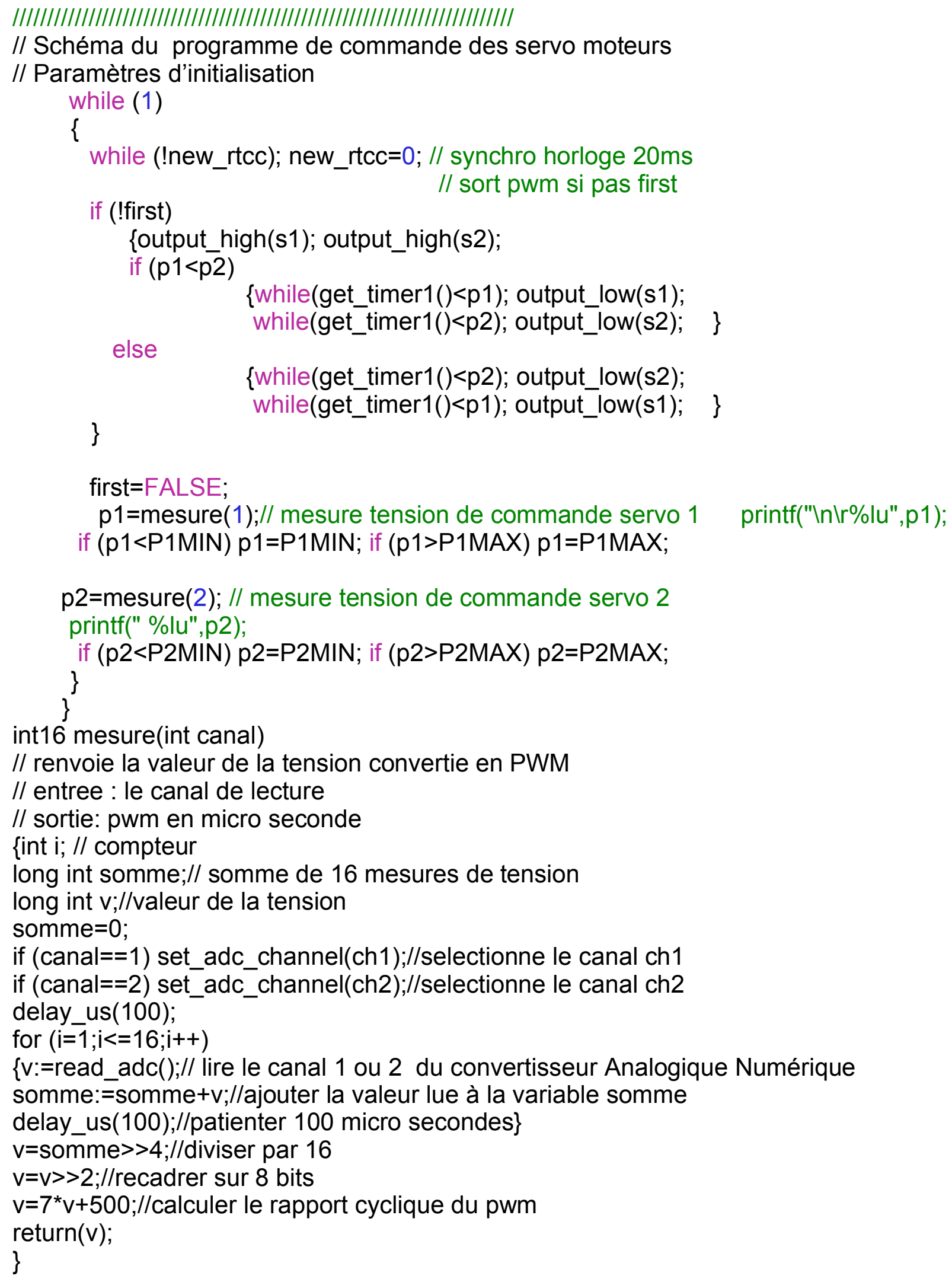

